\title{
Lepton angular distributions of fixed-target Drell-Yan experiments in perturbative QCD and a geometric approach
}

\author{
Wen-Chen Chang, ${ }^{1}$ Randall Evan McClellan, ${ }^{2,3}$ Jen-Chieh Peng, ${ }^{3}$ and Oleg Teryaev ${ }^{4}$ \\ ${ }^{1}$ Institute of Physics, Academia Sinica, Taipei 11529, Taiwan \\ ${ }^{2}$ Thomas Jefferson National Accelerator Facility, Newport News, Virginia 23606, USA \\ ${ }^{3}$ Department of Physics, University of Illinois at Urbana-Champaign, Urbana, Illinois 61801, USA \\ ${ }^{4}$ Bogoliubov Laboratory of Theoretical Physics, JINR, 141980 Dubna, Russia
}

(Received 25 November 2018; published 24 January 2019)

\begin{abstract}
The lepton angular distributions of the Drell-Yan process in fixed-target experiments are investigated by NLO and NNLO perturbative QCD. We present the calculated angular parameters $\lambda, \mu, \nu$ and the degree of violation of the Lam-Tung relation, $1-\lambda-2 \nu$, for the NA10, E615 and E866 experiments. Predictions for the ongoing COMPASS and SeaQuest experiments are also presented. The transverse momentum $\left(q_{T}\right)$ distributions of $\lambda$ and $\nu$ show a clear dependence on the dimuon mass $(Q)$ while those of $\mu$ have a strong rapidity $\left(x_{F}\right)$ dependence. Furthermore, $\lambda$ and $\nu$ are found to scale with $q_{T} / Q$. These salient features could be qualitatively understood by a geometric approach where the lepton angular distribution parameters are expressed in terms of the polar and azimuthal angles of the "natural axis" in the dilepton rest frame.
\end{abstract}

DOI: 10.1103/PhysRevD.99.014032

\section{INTRODUCTION}

The Drell-Yan (D-Y) process [1] is one of the important experimental approaches to explore the partonic structure of hadrons [2]. It is a unique tool for accessing the structures of unstable hadrons such as pions and kaons [3-5]. The D-Y process plays an essential role in probing the sea quarks of protons [6-8] as well. The transverse momentum $\left(q_{T}\right)$ distributions of the D-Y cross sections yield important information on the intrinsic transverse momentum $\left(k_{T}\right)$ distribution of partons [9] in the small- $q_{T}$ region. Furthermore, the polar and azimuthal angular distributions of leptons produced in unpolarized D-Y process are sensitive to the underlying reaction mechanisms and to novel parton distributions such as Boer-Mulders functions [10]. For measurement with a transversely polarized target, a recent experiment extracted information on Sivers functions for the first time via the D-Y process [11].

In the rest frame of the virtual photon in the D-Y process, a commonly used expression for the lepton angular distributions is given as [12]

$\frac{d \sigma}{d \Omega} \propto 1+\lambda \cos ^{2} \theta+\mu \sin 2 \theta \cos \phi+\frac{\nu}{2} \sin ^{2} \theta \cos 2 \phi$,

Published by the American Physical Society under the terms of the Creative Commons Attribution 4.0 International license. Further distribution of this work must maintain attribution to the author(s) and the published article's title, journal citation, and DOI. Funded by SCOAP ${ }^{3}$. where $\theta$ and $\phi$ refer to the polar and azimuthal angles of $l^{-}$ $\left(e^{-}\right.$or $\left.\mu^{-}\right)$. At leading-order (LO), $q \bar{q} \rightarrow \gamma^{*}$ with collinear partons leads to a transversely polarized virtual photon with a prediction of $\lambda=1$ and $\mu=\nu=0$ [1]. To describe the $\mathrm{D}-\mathrm{Y}$ process with finite $q_{T}$, higher-order QCD processes, such as $q \bar{q} \rightarrow \gamma^{*} G$ and $q G \rightarrow \gamma^{*} q$ in $\mathcal{O}\left(\alpha_{S}\right)$, should be included and these processes could alter the angular coefficients $\lambda, \mu$ and $\nu$ in principle. While $\lambda$ can now deviate from 1 , and $\mu$ and $\nu$ can be nonzero, a well-known result is that the Lam-Tung (L-T) relation [13],

$$
1-\lambda-2 \nu=0,
$$

holds for both NLO processes. Deviation from the L-T relation appears in the NNLO process $\mathcal{O}\left(\alpha_{S}^{2}\right)$ and beyond, e.g., $q \bar{q} \rightarrow \gamma^{*} G G, q G \rightarrow \gamma^{*} q G$ and $G G \rightarrow \gamma^{*} G$ according to $\mathrm{pQCD}$ [14].

Violation of the L-T relation was observed in the fixedtarget experiments with pion beams by NA10 [3] and E615 [4], while L-T was found to be satisfied in the D-Y production with proton beams by E866 [15]. The $q_{T}$ range of these fixed-target experiments is between 0 and $5 \mathrm{GeV}$. As for the measurements of $Z$ boson production in the collider experiments, CDF data of $p-\bar{p}$ collision [16] are consistent with the L-T relation, while CMS and ATLAS data of $p-p$ collision $[17,18]$ show a clear violation. The violation of the L-T relation at $q_{T}>5 \mathrm{GeV}$ could be well described taking into account NNLO pQCD effect [19]. Lambersten and Vogelsang [20] compared the NLO and NNLO pQCD calculations of $\lambda$ and $\nu$ with the data of fixedtarget experiments NA10, E615 and E866. Overall the 
agreement is not as good as seen in the collider data at large $q_{T}$.

Recently we interpreted the violation of the L-T relation as a consequence of the acoplanarity of the partonic subprocess [21,22]. This acoplanarity can arise from intrinsic transverse momenta of partons inside the hadrons, or from the perturbative gluon radiation beyond $\mathcal{O}\left(\alpha_{s}\right)$ such that the axis of the annihilating quark-antiquark pair (natural axis) no longer necessarily resides on the colliding hadron plane. In addition to the violation of the L-T relation, other salient features of the $q_{T}$ dependence of the $\lambda, \mu$ and $\nu$ parameters of the $Z$ production data from the collider experiments [21,22], as well as the rotational invariance properties of these parameters [23], could be well explained by this intuitive geometric approach.

In this work we compare the $\lambda, \mu, \nu$ data measured at NA10 [3], E615 [4] and E866 [15] with the fixed-order pQCD calculations. The approach is similar to what was done in Ref. [20], but we extend the study to include the L-T violation quantity $1-\lambda-2 \nu$, the $\mu$ parameter, as well as the scaling behavior of these angular parameters. Furthermore we present the NLO pQCD predictions for the ongoing COMPASS [24] and SeaQuest [25] experiments on the dimuon mass $Q$ and Feynman- $x\left(x_{F}\right)$ dependence of the angular parameters. The common features between the pQCD and the geometric approach [21,22] are also discussed.

This paper is organized as follows. In Sec. II, we describe how the fixed-order pQCD calculation is performed to extract the angular distribution parameters. The results from the pQCD calculations for the existing and forthcoming fixed-target experiments are then presented in Secs. III and IV, respectively. We further interpret some notable features of pQCD results using the geometric model in Sec. V, followed by conclusion in Sec. VI.

\section{CALCULATIONS OF ANGULAR PARAMETERS IN DYNNLO}

The formalism of the NLO $\left(\mathcal{O}\left(\alpha_{S}\right)\right)[26]$ and the NNLO $\left(\mathcal{O}\left(\alpha_{S}^{2}\right)\right)$ [27] QCD of the D-Y process have been known for a while. It is not until recently that packages of evaluating the differential D-Y cross sections up to $\mathcal{O}\left(\alpha_{s}^{2}\right)$ from $p-p$ and $p-\bar{p}$ collisions are available for public usage: DYNNLO [28] and FEWZ [29]. Both packages are parton-level Monte Carlo programs and they provide the differential cross sections for the $\mathrm{D}-\mathrm{Y}$ process and $W / Z$ vector boson production. The threshold resummation of soft-gluon emission at small $q_{T}$ is not included in these two packages. As discussed in Ref. [20], even though resummation is important for the cross sections, it is expected not to affect the angular parameters [30,31].

In this work we utilize the DYNNLO (version 1.5) package [32]. With some minor modifications, the code can evaluate the D-Y cross sections induced by pion or proton beams on proton or neutron targets. Via the LHAPDF6 framework [33], the parton distribution functions (PDFs) [34] used for the protons and neutrons are "CT14nlo" and "CT14nnlo" in the NLO and NNLO calculations, respectively, and "GRVPI1" for the pion PDFs in both NLO and NNLO calculations. The factorization scale $\left(\mu_{F}\right)$ and renormalization scale $\left(\mu_{R}\right)$ are set as $\mu_{F}=\mu_{R}=Q$.

In order to calculate the $\lambda, \mu$, and $\nu$ parameters, we first calculate the $A_{i}$ parameters in an alternative expression of the lepton angular distributions of the $\mathrm{D}-\mathrm{Y}$ process as follows [35]:

$$
\begin{aligned}
\frac{d \sigma}{d \Omega} \propto & \left(1+\cos ^{2} \theta\right)+\frac{A_{0}}{2}\left(1-3 \cos ^{2} \theta\right) \\
& +A_{1} \sin 2 \theta \cos \phi+\frac{A_{2}}{2} \sin ^{2} \theta \cos 2 \phi
\end{aligned}
$$

where $\theta$ and $\phi$, same as in Eq. (1), are the polar and azimuthal angles of $l^{-}\left(e^{-}\right.$or $\left.\mu^{-}\right)$in the rest frame of $\gamma^{*}$. The angular coefficients $A_{i}$ could be evaluated by the moments of harmonic polynomial expressed as $[18,19]$

$$
\begin{aligned}
& A_{0}=4-10\left\langle\cos ^{2} \theta\right\rangle, \\
& A_{1}=5\langle\sin 2 \theta \cos \phi\rangle, \\
& A_{2}=10\left\langle\sin ^{2} \theta \cos 2 \phi\right\rangle,
\end{aligned}
$$

where $\langle f(\theta, \phi)\rangle$ denotes the moment of $f(\theta, \phi)$, i.e., the weighted average of $f(\theta, \phi)$ by the cross sections in Eq. (3). It is straightforward to show that $\lambda, \mu, \nu$ in Eq. (1) are related to $A_{0}, A_{1}, A_{2}$ via

$$
\lambda=\frac{2-3 A_{0}}{2+A_{0}} ; \quad \mu=\frac{2 A_{1}}{2+A_{0}} ; \quad \nu=\frac{2 A_{2}}{2+A_{0}} .
$$

Equation (5) shows that the $\mathrm{L}-\mathrm{T}$ relation, $1-\lambda-2 \nu=0$, is equivalent to $A_{0}=A_{2}$.

\section{COMPARISON WITH EXISTING DATA FROM NA10, E615 AND E866}

Now we compare the results of $\lambda, \mu, \nu$, and the L-T violation, $1-\lambda-2 \nu$, from the fixed-order $\mathrm{pQCD}$ calculations with existing data from fixed-target experiments. The angular parameters are evaluated as a function of the dimuon's $q_{T}$ in the Collins-Soper frame [35]. We first consider the data from NA10 [3] and E615 [4] for $\pi^{-}$beam interacting with tungsten $(W)$ targets. The NA10 experiment used three different beam energies: 140, 194 and $286 \mathrm{GeV}$, while E615 utilized a single beam energy of $252 \mathrm{GeV}$. Since the experiments were done with tungsten targets, the cross sections per nucleon were calculated by the weighted average of the $\pi^{-} p$ and $\pi^{-} n$ cross sections with 74 protons and 110 neutrons. Following the experimental acceptance specified in Ref. [20], we apply the kinematic cuts listed in Table I. The results of NLO (red 
TABLE I. Kinematic cuts applied for the experimental acceptance in the fixed-order pQCD calculation.

\begin{tabular}{lccc}
\hline \hline Experiment & $\mathrm{Q}(\mathrm{GeV})$ & $x_{1}$ & $x_{F}$ \\
\hline NA10 & $4.05 \leq Q \leq 8.55$ & $0 \leq x_{1} \leq 0.7$ & $0 \leq x_{F}$ \\
E615 & $4.05 \leq Q \leq 8.55$ & $0.2 \leq x_{1} \leq 1$ & $0 \leq x_{F}$ \\
E866 & $4.5 \leq Q \leq 15^{\mathrm{a}}$ & $0 \leq x_{1} \leq 0.7$ & $0 \leq x_{F}$ \\
\hline \hline
\end{tabular}

${ }^{\mathrm{a}}$ Excluding the $\Upsilon$ region $9 \leq Q \leq 10.7 \mathrm{GeV}$.

points) and NNLO (blue points) calculations together with the measurements (black points) are shown in Figs. 1-4.

Overall, the calculated $\lambda, \mu$ and $\nu$ exhibit distinct $q_{T}$ dependencies. At $q_{T} \rightarrow 0, \lambda, \mu$ and $\nu$ approach the values

\section{NA10 $\pi^{-}+W$ at $140 \mathrm{GeV}$}
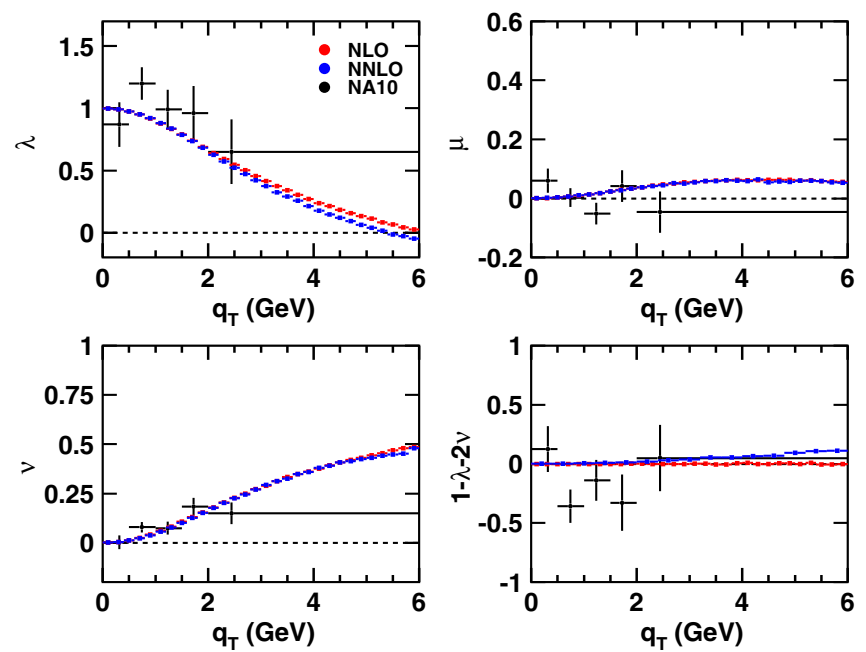

FIG. 1. Comparison of NLO (red points) and NNLO (blue points) fixed-order pQCD calculations with the NA10 $\pi^{-}+W$ D-Y data at $140 \mathrm{GeV}$ [3] (black points) for $\lambda, \mu, \nu$ and $1-\lambda-2 \nu$.

NA10 $\pi^{-}+\mathrm{W}$ at $194 \mathrm{GeV}$
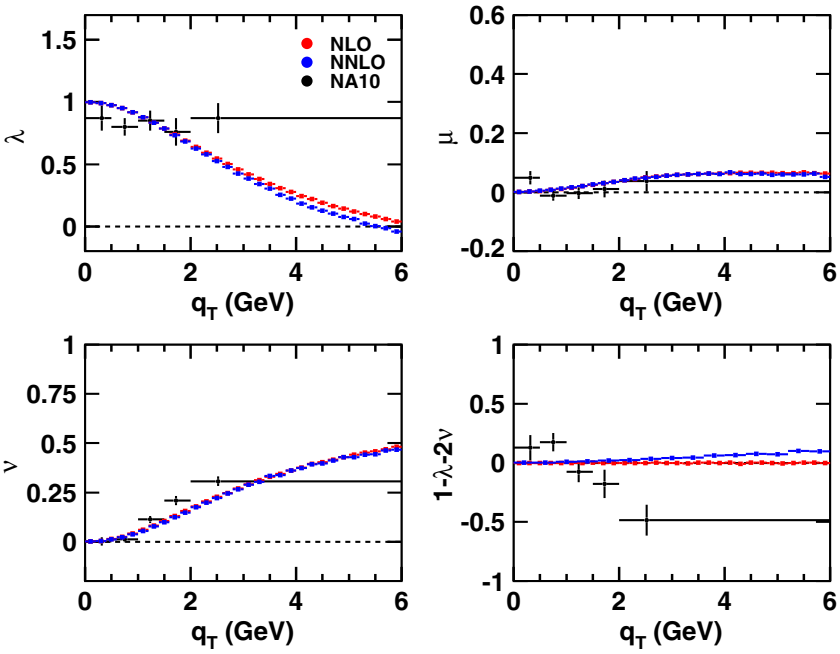

FIG. 2. Same as Fig. 1, but for NA10 data [3] with 194-GeV $\pi^{-}$ beam. predicted by the collinear parton model [1]: $\lambda=1$ and $\mu=\nu=0$. As $q_{T}$ increases, Figs. 1-4 show that $\lambda$ decreases toward its large $-q_{T}$ limit of $-1 / 3$ while $\nu$ increases toward $2 / 3$, for both $q \bar{q}$ and $q G$ processes shown in Ref. [21]. The $q_{T}$ dependence of $\mu$ is relatively mild compared to $\lambda$ and $\nu$. This is understood as a result of some cancellation effect, to be discussed in Sec. V. Comparing the results of the NLO with the NNLO calculation, $\lambda(\mathrm{NNLO})$ is smaller than $\lambda$ (NLO) while $\mu$ and $\nu$ are very similar for NLO and NNLO. The L-T violation, $1-\lambda-2 \nu$, is zero in the NLO calculation, and turns to be nonzero and positive in the NNLO calculation.

As shown in Figs. 1-4, while some general features of the NA10 and E615 data are described by the pQCD

\section{NA10 $\pi^{-}+\mathrm{W}$ at $286 \mathrm{GeV}$}
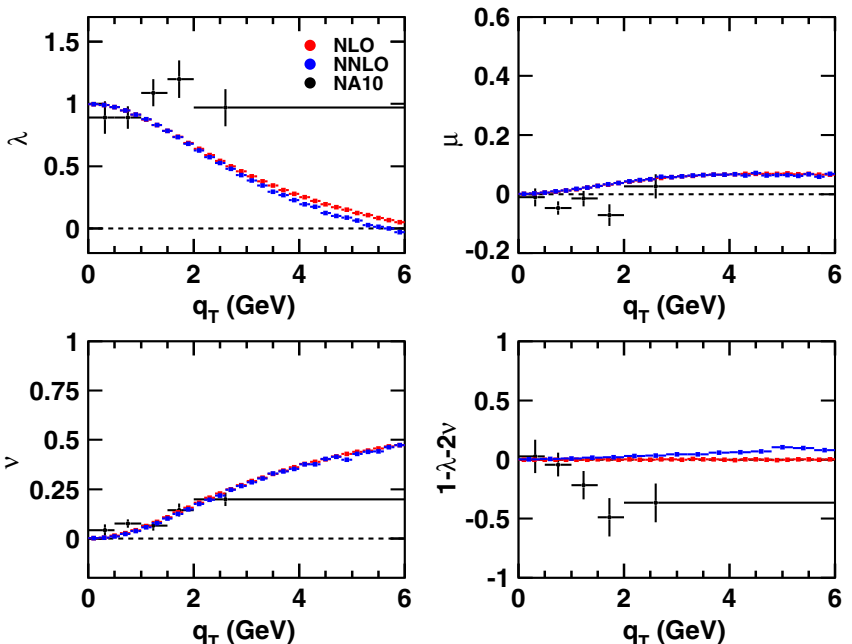

FIG. 3. Same as Fig. 1, but for NA10 data [3] with $286-\mathrm{GeV} \pi^{-}$ beam.

$\mathrm{E} 615 \pi^{-}+\mathrm{W}$ at $252 \mathrm{GeV}$
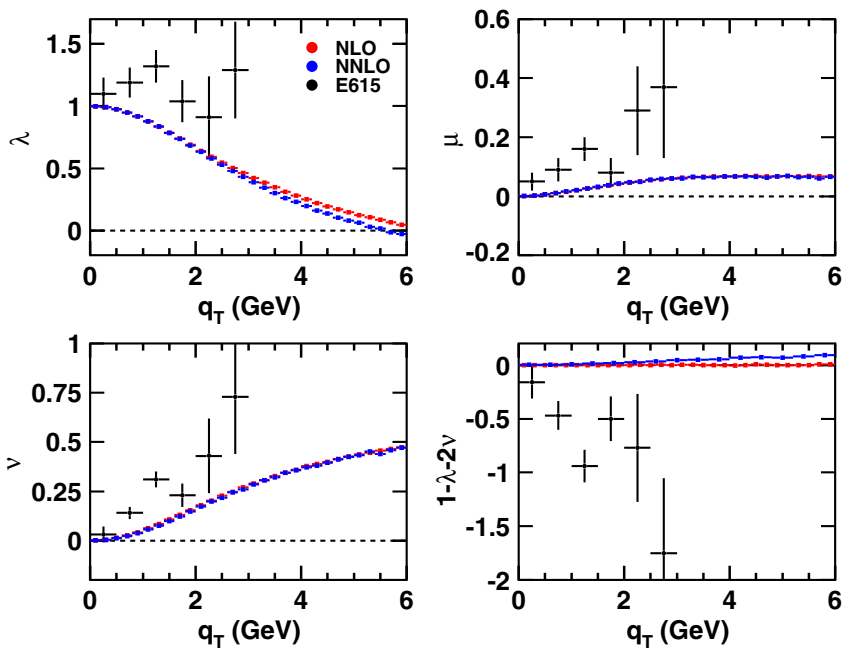

FIG. 4. Comparison of NLO (red points) and NNLO (blue points) fixed-order pQCD calculations with the E615 $\pi^{-}+W$ D-Y data at $252 \mathrm{GeV}$ [4] (black points) for $\lambda, \mu, \nu$ and $1-\lambda-2 \nu$. 
calculations, there are notable differences between the data and calculations. From the comparison between them, we find:

(1) Perturbative QCD predicts that $\lambda$ drops as $q_{T}$ increases, but the data do not show this trend. The expected upper bound of $\lambda,|\lambda| \leq 1$, is sometimes exceeded by the data [20]. This could reflect the presence of some systematic uncertainties in the data.

(2) The agreement between the data and the pQCD calculation for the $\mu$ parameter is quite reasonable for NA10, but less so for E615.

(3) The increase of $\nu$ with $q_{T}$ observed in the NA10 data is in good agreement with the pQCD calculation. However, the E615 data are significantly higher than the calculation.

(4) The amount of the L-T violation, $1-\lambda-2 \nu$, for the data is much larger than the prediction from the NNLO pQCD. Moreover, the sign of this violation is negative for the data, but positive for the $\mathrm{pQCD}$. This apparent discrepancy could be partly caused by the unphysical values of $\lambda$ from the data, as $\lambda$ should not exceed 1.

Regarding these findings two remarks are in order. First, pQCD predicts a sizable magnitude for $\nu$, comparable to the data. Therefore, in order to extract the value of the nonperturbative Boer-Mulders function from the measured data of $\nu[10,36,37]$, contributions from the pQCD effect must be taken into account. Second, the pQCD calculation for $\mu$ tends to overestimate the NA10 data but underestimate the E615 data. As we will see in Sec. IV, $\mu$ has a strong dependence on $x_{F}$. The incomplete information on the $x_{F}$ acceptance of the experiments needed for the calculation could contribute to the discrepancy.

The $q_{T}$ dependencies of the angular distribution parameters of $800-\mathrm{GeV} p+p$ and $p+d \mathrm{D}-\mathrm{Y}$ are calculated and compared with the E866 measurements [15] in Figs. 5 and 6. Given the large experimental uncertainty, the $p+p$ data in Fig. 5 are not in disagreement with the calculation. For Fig. 6, where the $p+d$ data have smaller uncertainties, the agreement between data and the calculation is rather poor. In particular, the data on $\lambda$ are in general larger than 1 , violating the expected upper bound for $\lambda[20]$.

That the $\nu$ data are less than the $\mathrm{pQCD}$ prediction in Figs. 5 and 6, suggests a negative contribution from the Boer-Mulders effect in the proton-induced DY. This is opposite to the situation in Fig. 4, where the $\nu$ data are more positive than the $\mathrm{pQCD}$, suggesting a positive contribution from the Boer-Mulders function in the pion-induced DY. Since the contribution of the Boer-Mulders effect in $\nu$ is proportional to the product of the individual Boer-Mulders functions of quarks and anti-quarks in the colliding hadrons, the proton D-Y data would imply that the seaquark Boer-Mulders function has a sign opposite to that of
$\mathrm{E} 866 \mathrm{p}+\mathrm{p}$ at $800 \mathrm{GeV}$
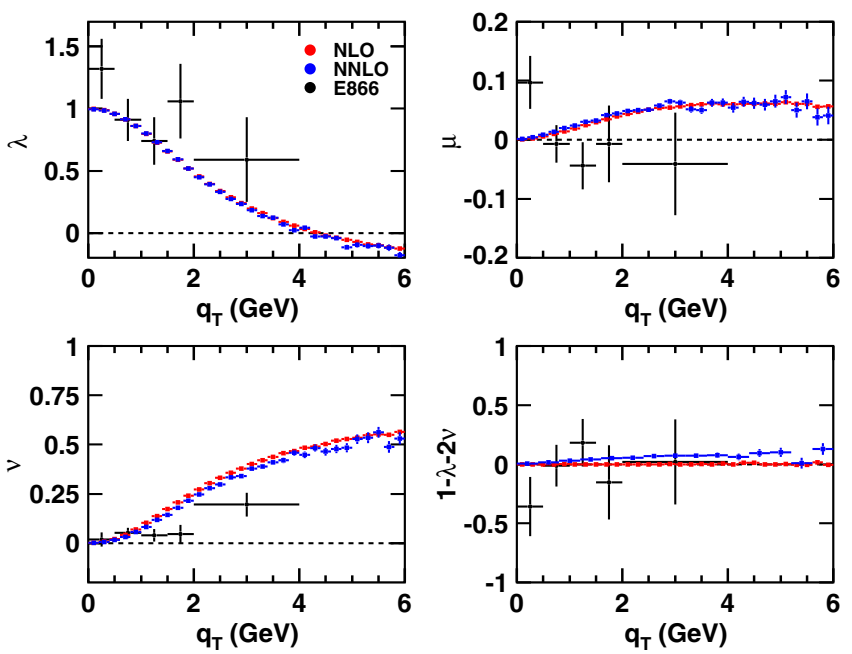

FIG. 5. Comparison of NLO (red points) and NNLO (blue points) fixed-order $\mathrm{pQCD}$ calculations with the $\mathrm{E} 866 p+p \mathrm{D}-\mathrm{Y}$ data at $800 \mathrm{GeV}$ [15] (black points) for $\lambda, \mu, \nu$ and $1-\lambda-2 \nu$.

E866 p+d at $800 \mathrm{GeV}$
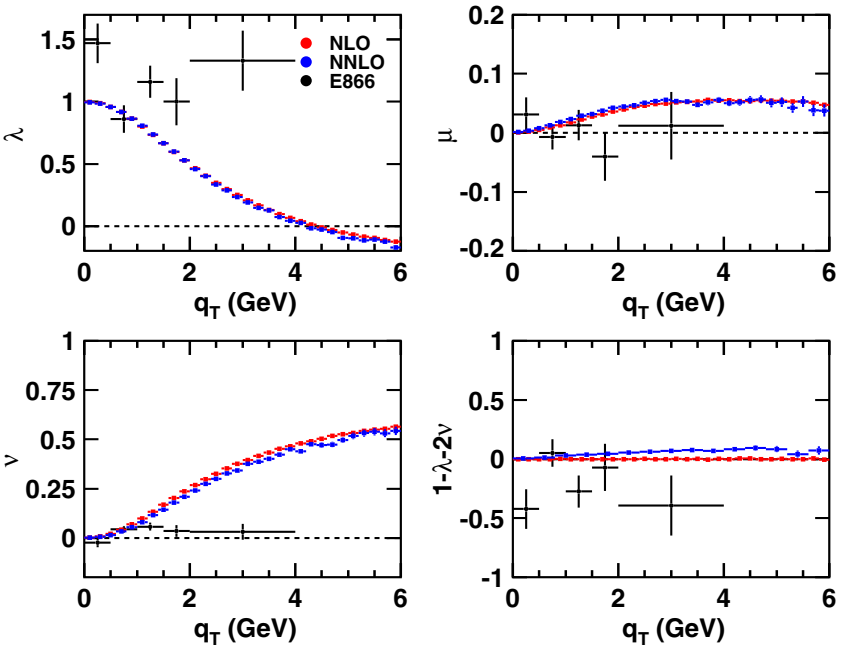

FIG. 6. Same as Fig. 5, but for E866 data [15] with a liquid deuterium target.

the valence Boer-Mulders function in the proton [38]. The pion data from Fig. 4 suggests that the pion valence BoerMulders function has the same sign as the proton valence Boer-Mulders function [38].

The NNLO calculations predict a positive $1-\lambda-2 \nu$ at NNLO while the data are consistent with zero for the proton target and slightly negative for the deuteron one. The negative values of $1-\lambda-2 \nu$ for $p+d$ data are similar to the case for the pion D-Y data shown in Figs. 1-4. In Sec. V, we will discuss why $1-\lambda-2 \nu$ must be positive from the perspective of a geometric approach. 


\section{PQCD CALCULATIONS FOR THE COMPASS AND SEAQUEST EXPERIMENTS}

There are two ongoing fixed-target D-Y experiments which have collected new data on the lepton angular distributions. The first one is the COMPASS experiment at CERN [24], running with $190-\mathrm{GeV} \pi^{-}$beam and transversely-polarized $\mathrm{NH}_{3}$ target and unpolarized aluminum $(A l)$ and tungsten $(W)$ nuclear targets. The transversemomentum-dependent Sivers asymmetry in the polarized D-Y process was reported recently [11], and high-statistics unpolarized D-Y data on the $W$ target have also been collected. The second one is the SeaQuest experiment at Fermilab [25], aiming at the measurement of $\bar{d}(x) / \bar{u}(x)$ ratio at intermediate- $x$ region via the D-Y process. It has taken data with the $120-\mathrm{GeV}$ proton beam on unpolarized hydrogen, deuterium and various nuclear targets. Both COMPASS and SeaQuest experiments have collected data on the lepton angular distributions of the D-Y process. The final results are expected to be available in the near future. In addition, the extension of the SeaQuest experiment, the E1039 experiment [39], expects to take more data relevant to the angular distributions in the near future.

Here we present the results of the angular coefficients $\lambda$, $\mu$ and $\nu$ as a function of $q_{T}$ in various bins of $Q$ and $x_{F}$. There are three bins for $Q$ in the range of $4.0-7.0 \mathrm{GeV}$, as well as three bins for $x_{F}$ in the range of $0-0.6$. These results could be convoluted by the COMPASS and SeaQuest spectrometer acceptances later for a direct comparison with experimental data. Since there are no significant difference between the NLO and NNLO results, we present only the results from the NLO calculation to illustrate the major features.

The mean values of $Q$ and $x_{F}$ in each bin are listed in Tables II and III. The pQCD calculations show that the $q \bar{q}$

TABLE II. Mean values of $Q$ and $x_{F}$ in each $Q$ bin calculated for COMPASS and SeaQuest.

\begin{tabular}{lccccc}
\hline \hline & \multicolumn{2}{c}{ COMPASS } & & \multicolumn{2}{c}{ SeaQuest } \\
\cline { 2 - 3 } \cline { 5 - 6 } Bin & $\langle Q\rangle(\mathrm{GeV})$ & $\left\langle x_{F}\right\rangle$ & & $\langle Q\rangle(\mathrm{GeV})$ & $\left\langle x_{F}\right\rangle$ \\
\hline$Q=4-5 \mathrm{GeV}$ & 4.42 & 0.32 & & 4.36 & 0.24 \\
$Q=5-6 \mathrm{GeV}$ & 5.43 & 0.32 & & 5.36 & 0.23 \\
$Q=6-7 \mathrm{GeV}$ & 6.43 & 0.32 & & 6.36 & 0.22 \\
\hline \hline
\end{tabular}

TABLE III. Mean values of $Q$ and $x_{F}$ in each $x_{F}$ bin calculated for COMPASS and SeaQuest.

\begin{tabular}{lccccc}
\hline \hline & \multicolumn{2}{c}{ COMPASS } & & \multicolumn{2}{c}{ SeaQuest } \\
\cline { 2 - 3 } \cline { 5 - 6 } Bin & $\langle Q\rangle(\mathrm{GeV})$ & $\left\langle x_{F}\right\rangle$ & & $\langle Q\rangle(\mathrm{GeV})$ & $\left\langle x_{F}\right\rangle$ \\
\hline$x_{F}=0.0-0.2$ & 5.01 & 0.10 & & 4.56 & 0.10 \\
$x_{F}=0.2-0.4$ & 5.06 & 0.30 & & 4.55 & 0.29 \\
$x_{F}=0.4-0.6$ & 5.10 & 0.49 & & 4.54 & 0.48 \\
\hline \hline
\end{tabular}

process dominates over the whole $q_{T}$ region for the $\pi^{-}$induced COMPASS experiment while the $q G$ process becomes more important for $q_{T}>1 \mathrm{GeV}$ in the protoninduced SeaQuest experiment. Through this study, the $Q$ - and $x_{F}$-dependencies of $\lambda, \mu$ and $\nu$ are also investigated.

Figures 7 and 8 show $\lambda, \mu$ and $\nu$ as a function of $q_{T}$ for various bins of $Q$ and $x_{F}$. The $q_{T}$ distributions of $\lambda$ and $\nu$ parameters depend sensitively on $Q$, but only weakly on $x_{F}$. As for $\mu$, its $q_{T}$ distribution has strong dependencies on $x_{F}$ and on $Q$. In particular, the magnitude of $\mu$ is small when $x_{F}$ is close to 0 and its sign could even turn negative at some $q_{T}$ region. As $x_{F}$ increases, the magnitude of $\mu$ increases pronouncedly.

COMPASS $\pi^{-}+\mathrm{W}$ at $190 \mathrm{GeV}$

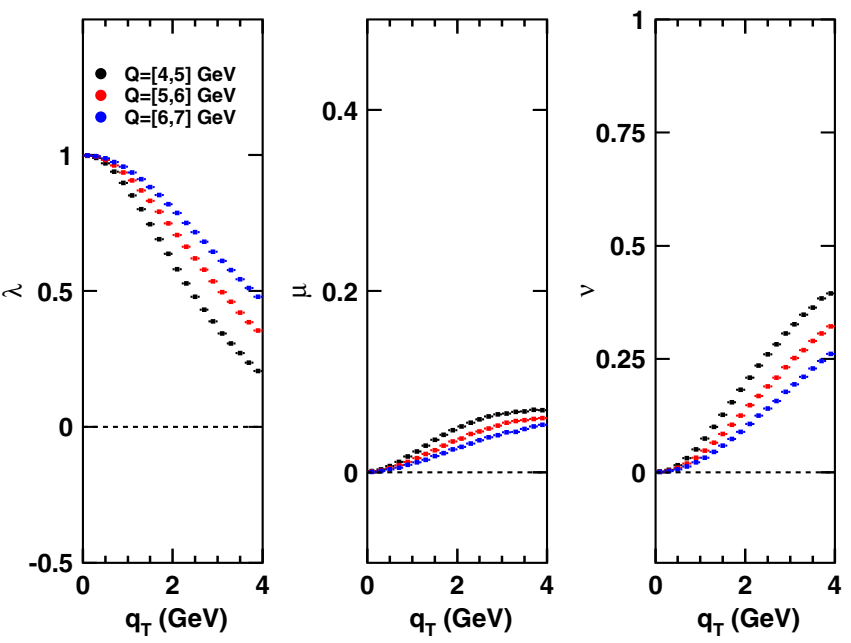

(a)

COMPASS $\pi^{-}+\mathrm{W}$ at $190 \mathrm{GeV}$
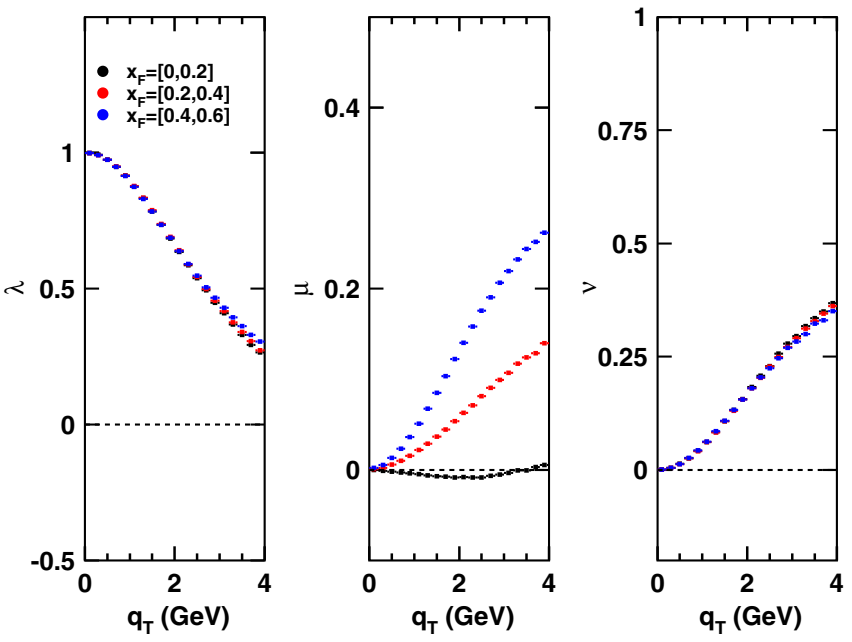

(b)

FIG. 7. (a) NLO pQCD results of $\lambda$, $\mu$, and $\nu$ as a function of $q_{T}$ at several $Q$ bins and $x_{F}>0$ for D-Y production off the tungsten target with $190-\mathrm{GeV} \pi^{-}$beam in the COMPASS experiment. (b) Same as (a) but at several $x_{F}$ bins and $4<Q<9 \mathrm{GeV}$. 
SeaQuest $p+p$ at $120 \mathrm{GeV}$
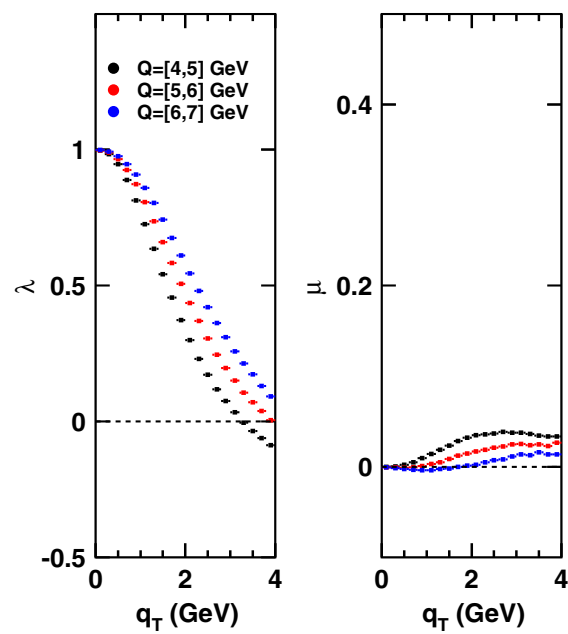

(a)

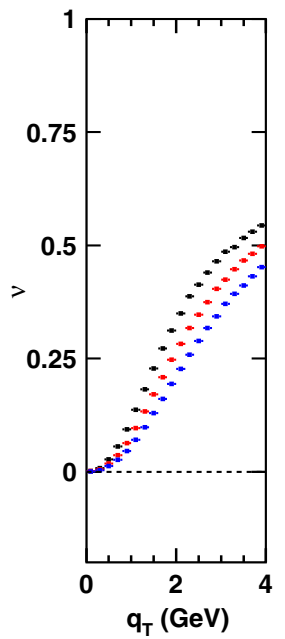

SeaQuest p+p at $120 \mathrm{GeV}$
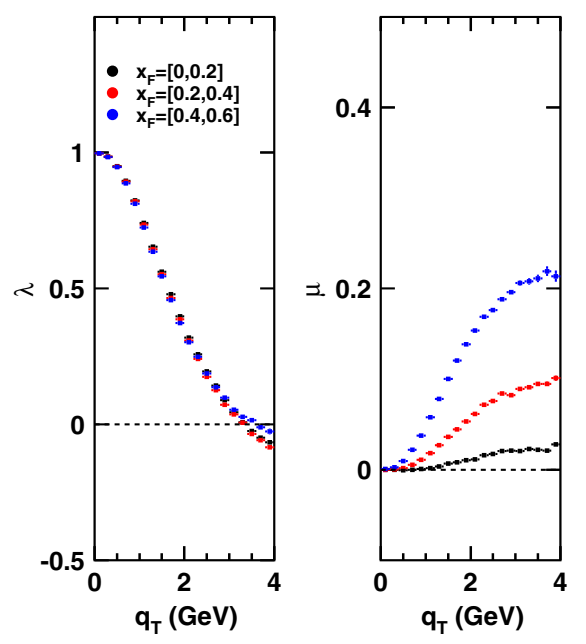

(b)

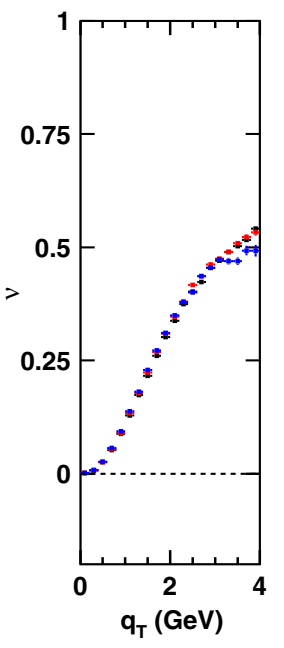

$q_{T}(\mathrm{GeV})$

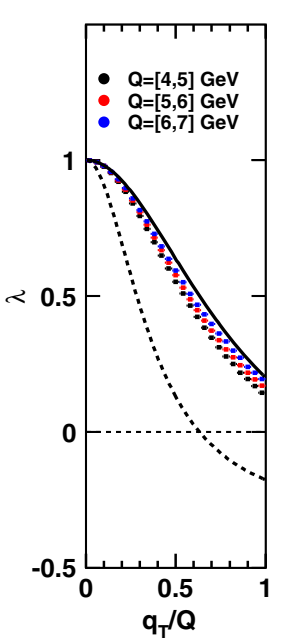

COMPASS $\pi^{-}+\mathrm{W}$ at $190 \mathrm{GeV}$

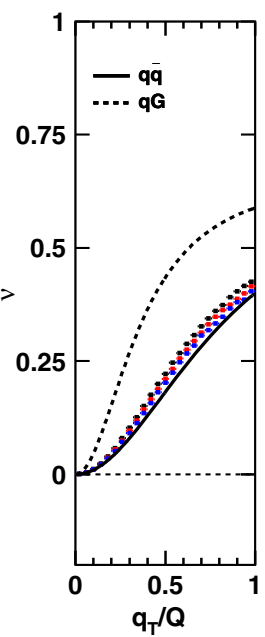

(a)

\section{COMPASS $\pi^{-}+\mathrm{W}$ at $190 \mathrm{GeV}$}
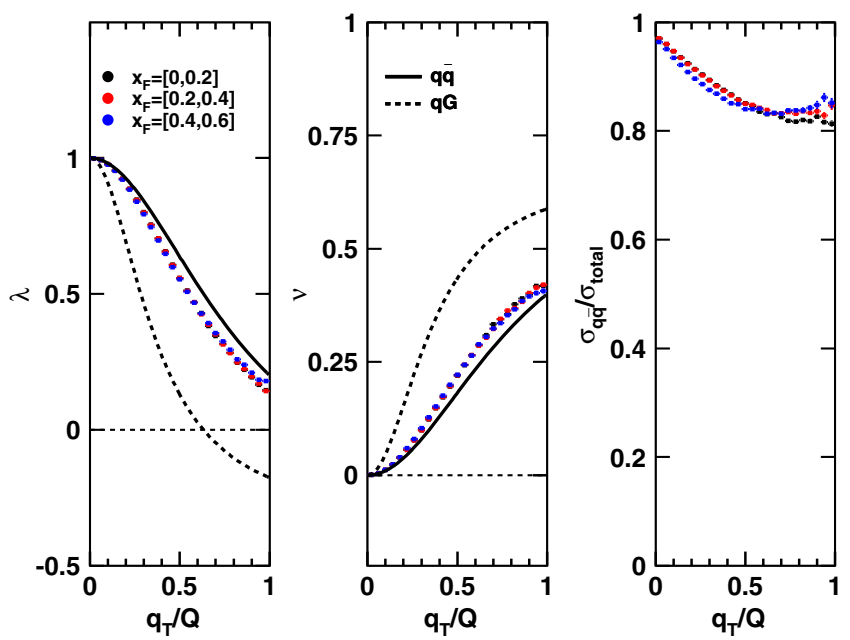

(b)

FIG. 8. (a) NLO pQCD results of $\lambda, \mu$, and $\nu$ as a function of $q_{T}$ at several $Q$ bins and $x_{F}>0$ for $\mathrm{D}-\mathrm{Y}$ production off the proton target with $120-\mathrm{GeV}$ proton beam in the SeaQuest experiment. (b) Same as (a) but at several $x_{F}$ bins and $4<Q<9 \mathrm{GeV}$.

In perturbative $\mathrm{QCD}$ at $\mathcal{O}\left(\alpha_{S}\right)$, ignoring the intrinsic transverse momenta of the colliding partons, the $\lambda$ and $\nu$ coefficients in the Collins-Soper frame for the $q \bar{q} \rightarrow \gamma^{*} G$ annihilation process $[30,31,40]$ and the $q G \rightarrow \gamma^{*} q$ Compton process $[3,41,42]$ are given as

$$
\begin{aligned}
& \lambda=\frac{2 Q^{2}-q_{T}^{2}}{2 Q^{2}+3 q_{T}^{2}} \quad \nu=\frac{2 q_{T}^{2}}{2 Q^{2}+3 q_{T}^{2}} \quad(q \bar{q}) \\
& \lambda=\frac{2 Q^{2}-5 q_{T}^{2}}{2 Q^{2}+15 q_{T}^{2}} \quad \nu=\frac{10 q_{T}^{2}}{2 Q^{2}+15 q_{T}^{2}} \quad(q G),
\end{aligned}
$$

where $q_{T}$ and $Q$ are the transverse momentum and mass, respectively, of the dilepton. While the expression for $q \bar{q} \rightarrow$ $\gamma^{*} G$ is exact, that for $q G \rightarrow \gamma^{*} q$ is obtained with some

FIG. 9. (a) NLO pQCD results of $\lambda, \nu$ and the fractions of $q \bar{q}$ process contribution in the total cross sections as a function of scaled transverse momentum $q_{T} / Q$ for $\mathrm{D}-\mathrm{Y}$ production off the nuclear tungsten target with $190-\mathrm{GeV} \pi^{-}$beam in the COMPASS experiment. The NLO pQCD expressions of $q \bar{q}$ and $q G$ processes are denoted by the solid and dashed lines respectively. (b) Same as (a) but at several $x_{F}$ bins and $4<Q<9 \mathrm{GeV}$.

approximation. Equation (6) shows that $\lambda$ and $\nu$ scale with the dimensionless $q_{T} / Q$ in these pQCD NLO expressions. Nevertheless there is no $q_{T} / Q$ scaling for the $\mu$ parameter in NLO pQCD.

Figures 9 and 10 show the NLO calculations of $\lambda$ and $\nu$ for COMPASS and SeaQuest as a function of the variable $q_{T} / Q$ in the various $Q$ and $x_{F}$ bins. The corresponding expressions for the $q \bar{q}$ and $q G$ processes in Eq. (6) are denoted by the solid and dashed lines. Comparing Figs. 9 and 10 with Figs. 7 and 8, the $\lambda$ and $\nu$ values for different $Q$ bins now converge into a common curve when they are 
SeaQuest $p+p$ at $120 \mathrm{GeV}$
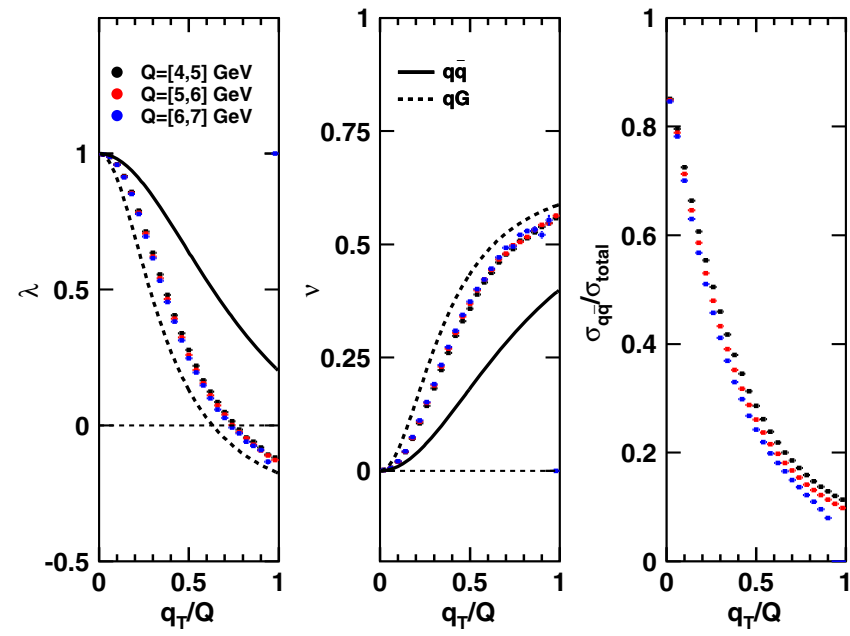

(a)

SeaQuest $p+p$ at $120 \mathrm{GeV}$

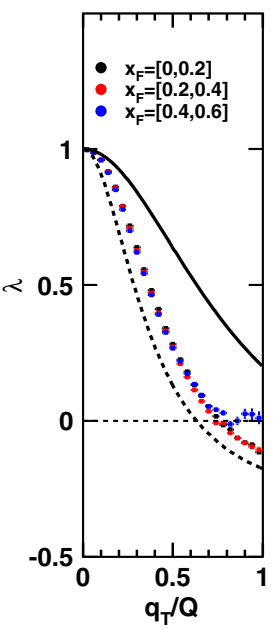

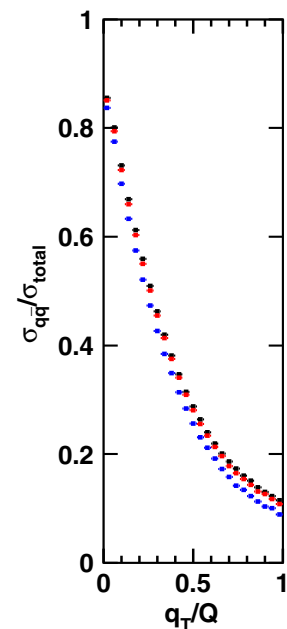

(b)

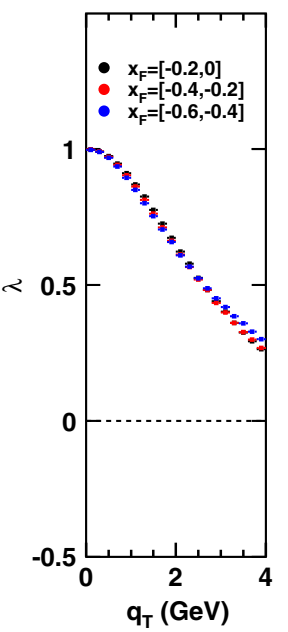

COMPASS $\pi^{-}+\mathrm{W}$ at $190 \mathrm{GeV}$

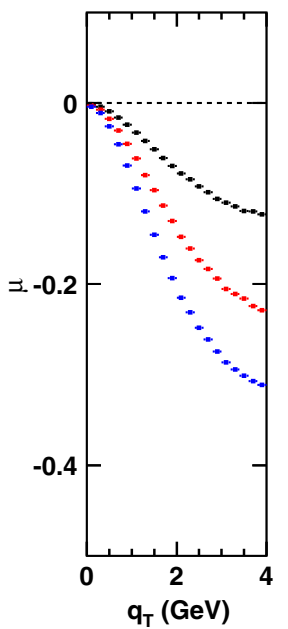

(a)

SeaQuest $p+p$ at $120 \mathrm{GeV}$
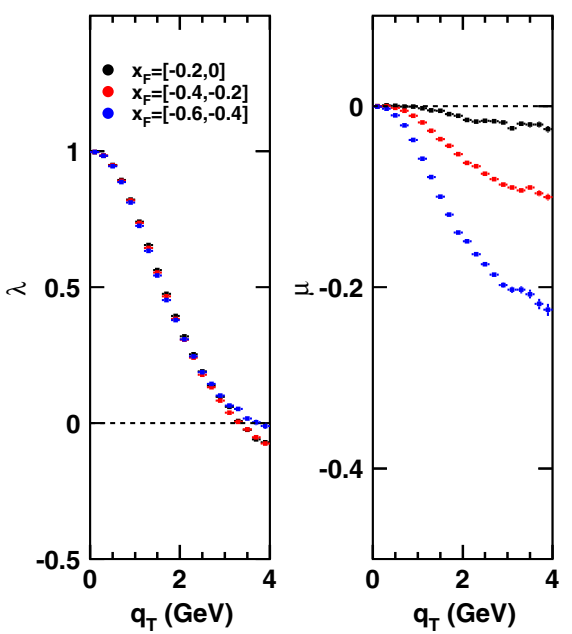

(b)
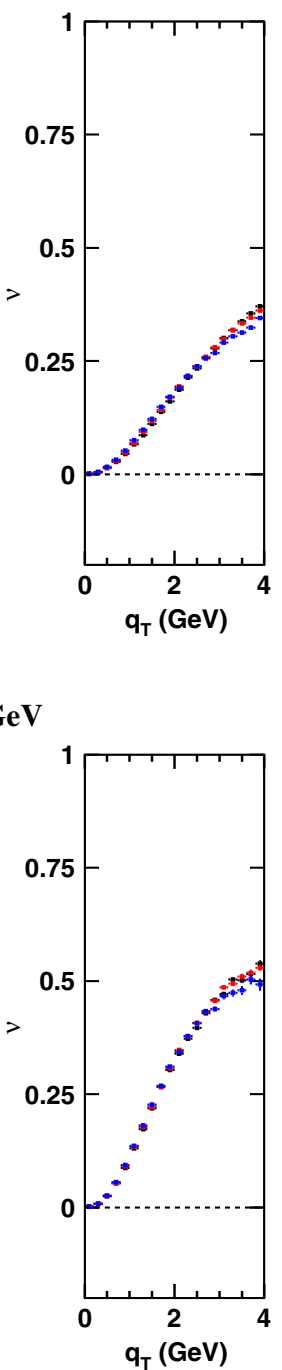

FIG. 10. (a) NLO pQCD results of $\lambda, \nu$ and the fractions of $q \bar{q}$ process contribution in the total cross sections as a function of scaled transverse momentum $q_{T} / Q$ for $\mathrm{D}-\mathrm{Y}$ production off the proton target with $120-\mathrm{GeV}$ proton beam in the SeaQuest experiment. The NLO pQCD expressions of $q \bar{q}$ and $q G$ processes are denoted by the solid and dashed lines respectively. (b) Same as (a) but at several $x_{F}$ bins and $4<Q<9 \mathrm{GeV}$. It is noted that the rough structure at large $q_{T} / Q$ region of the results for $x_{F}=$ 0.4-0.6 (blue points) is likely due to the fluctuation of calculations with $Q>7 \mathrm{GeV}$ near the edge of the phase space. The structure is expected to be removed, if one requires $Q<7 \mathrm{GeV}$ as the top figure.

plotted as a function of $q_{T} / Q$. This is consistent with the $q_{T} / Q$ scaling behavior of Eq. (6).

Figures 9 and 10 also display the fractions of the NLO cross sections due to the $q \bar{q}$ process for COMPASS and SeaQuest. The dominance of the $q \bar{q}$ process in the $\pi^{-}$induced D-Y at COMPASS explains why the $\mathrm{pQCD}$ results for $\lambda$ and $\nu$ are very close to the solid $q \bar{q}$ lines. In contrast,

FIG. 11. (a) NLO pQCD results of $\lambda, \mu$ and $\nu$ as a function of transverse momentum $q_{T}$ at several negative $x_{F}$ bins and $4<$ $Q<9 \mathrm{GeV}$ for D-Y production off the nuclear tungsten target with $190-\mathrm{GeV}$ proton beam in COMPASS experiment. (b) Same results of (a) for D-Y production off the proton target with 120$\mathrm{GeV}$ proton beam in SeaQuest experiment.

the proton-induced D-Y in SeaQuest has large contributions from the $q G$ process, resulting in the $\lambda$ and $\nu$ closer to the dashed $q G$ lines.

In comparison, we plot the $q_{T}$ distributions of $\lambda, \mu$ and $\nu$ in the negative $x_{F}(-0.6-0)$ for COMPASS and SeaQuest in Fig. 11. The $\lambda$ and $\nu$ remain the same as that in $x_{F}>0$ while $\mu$ turns mostly negative.

\section{GEOMETRIC MODEL}

As seen above, the existing D-Y data of lepton angular distributions can be reasonably well described by the NLO and NNLO pQCD calculations. Various salient features of 
$Q$ and $x_{F}$ dependencies as well as $q_{T} / Q$ scaling are observed in the predicted results of $\lambda, \mu$ and $\nu$ parameters for COMPASS and SeaQuest experiments based on NLO pQCD. It is of interest to check if these features could be understood using the geometric approach developed in Refs. [21,22].

Here we sketch the geometric approach of Refs. [21,22]. As illustrated in Fig. 12, we define three different planes, the hadron plane, the quark plane, and the lepton plane, in the Collins-Soper frame. In the $\gamma^{*}$ rest frame, the beam and target hadron momenta, $\vec{P}_{B}$ and $\vec{P}_{T}$ form the "hadron plane" on which the $\hat{z}$ axis, bisecting the $\vec{P}_{B}$ and $-\vec{P}_{T}$ vectors, lies. A pair of collinear $q$ and $\bar{q}$ with equal momenta annihilate into a $\gamma^{*}$. The momentum unit vector of $q$ is defined as $\hat{z}^{\prime}$, and the "quark plane" is formed by the $\hat{z}^{\prime}$ and $\hat{z}$ axes. Finally, the "lepton plane" is formed by the momentum vector of $l^{-}$and the $\hat{z}$ axis. The polar and azimuthal angles of the $\hat{z}^{\prime}$ axis in the Collins-Soper frame are denoted as $\theta_{1}$ and $\phi_{1}$. As shown in Refs. [21,22], the angular coefficients $A_{i}$ in Eq. (3) can be expressed in term of $\theta_{1}$ and $\phi_{1}$ as follows:

$$
\begin{aligned}
& A_{0}=\left\langle\sin ^{2} \theta_{1}\right\rangle \\
& A_{1}=\frac{1}{2}\left\langle\sin 2 \theta_{1} \cos \phi_{1}\right\rangle \\
& A_{2}=\left\langle\sin ^{2} \theta_{1} \cos 2 \phi_{1}\right\rangle .
\end{aligned}
$$

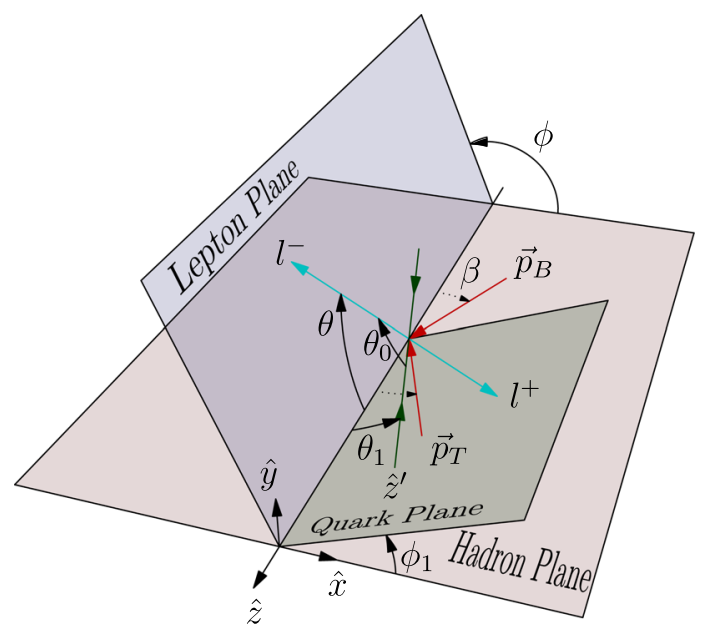

FIG. 12. Definition of the Collins-Soper frame and various angles and planes in the rest frame of $\gamma^{*}$. The hadron plane is formed by $\vec{P}_{B}$ and $\vec{P}_{T}$, the momentum vectors of the beam (B) and target (T) hadrons. The $\hat{x}$ and $\hat{z}$ axes of the Collins-Soper frame both lie in the hadron plane with the $\hat{z}$ axis bisecting the $\vec{P}_{B}$ and $-\vec{P}_{T}$ vectors. The quark $(q)$ and antiquark $(\bar{q})$ annihilate collinearly with equal momenta to form $\gamma^{*}$, while the quark momentum vector $\hat{z}^{\prime}$ and the $\hat{z}$ axis form the quark plane. The polar and azimuthal angles of $\hat{z}^{\prime}$ in the Collins-Soper frame are $\theta_{1}$ and $\phi_{1}$. The $l^{-}$and $l^{+}$are emitted back-to-back with $\theta$ and $\phi$ as the polar and azimuthal angles for $l^{-}$.
The $\langle\ldots\rangle$ in Eq. (7) is a reminder that the measured values of $A_{i}$ at a given kinematic bin are averaged over events having particular values of $\theta_{1}$ and $\phi_{1}$.

As discussed in Refs. [21,22], up to $\operatorname{NLO}\left(\mathcal{O}\left(\alpha_{S}\right)\right)$ in $\mathrm{pQCD}$, the quark plane coincides with the hadron plane and $\phi_{1}=0$. Therefore $A_{0}=A_{2}$ or $1-\lambda-2 \nu=0$, i.e., the L-T relation is satisfied. Higher order pQCD processes allow the quark plane to deviate from the hadron plane, i.e., $\phi_{1} \neq 0$, leading to the violation of the L-T relation. For a nonzero $\phi_{1}$, Eq. (7) shows that $A_{2}<A_{0}$. Therefore, when the $\mathrm{L}-\mathrm{T}$ relation is violated, $A_{0}$ must be greater than $A_{2}$ or, equivalently, $1-\lambda-2 \nu>0$. This expectation of $1-\lambda-2 \nu>0$ in the geometric approach is in agreement with the results of NNLO pQCD calculations shown in Figs. 1-6. The geometric approach offers a simple interpretation for this result.

Figures 7(b) and 8(b) show that the $q_{T}$ dependencies for $\lambda$ and $\nu$ are insensitive to the value of $x_{F}$. In contrast, the $\mu$ parameter depends sensitively on $x_{F}$. This striking difference between the $\lambda, \mu$ and $\nu$ parameters can be understood in the geometric approach. At the next-to-leading order (NLO), $\mathcal{O}\left(\alpha_{S}\right)$, a hard gluon or a quark (antiquark) is emitted so that $\gamma^{*}$ acquires nonzero $q_{T}$. Figure 13(a) shows a diagram for the $q-\bar{q}$ annihilation process in which a gluon is emitted from the quark in the beam hadron. In this case, the momentum vector of the quark is modified such

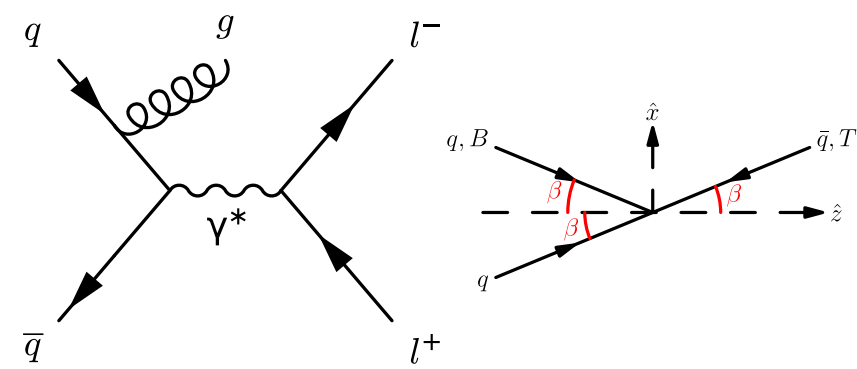

(a)

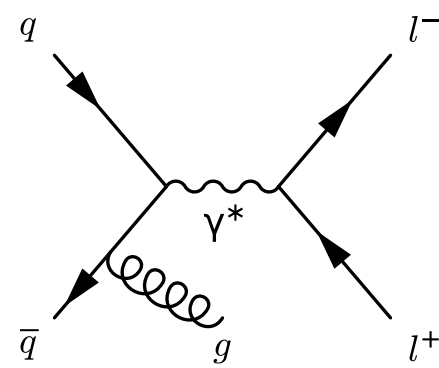

(c) (b)

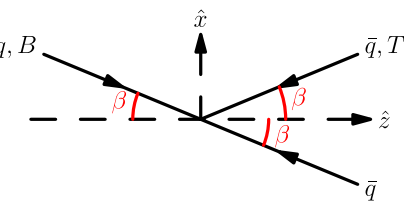

(d)
FIG. 13. (a) Feynman diagram for $q-\bar{q}$ annihilation where a gluon is emitted from a quark in the beam hadron. (b) Momentum vectors for $q$ and $\bar{q}$ in the $\mathrm{C}-\mathrm{S}$ frame before and after gluon emission. The momentum direction of $q$ is now collinear with that of $\bar{q}$. (c) Feynman diagram for the case where a gluon is emitted from an antiquark in the target hadron. (d) Momenta vectors for $q$ and $\bar{q}$ in the $\mathrm{C}-\mathrm{S}$ frame before and after gluon emission for diagram (c). 
TABLE IV. Angles $\theta_{1}$ and $\phi_{1}$ for four cases of gluon emission in the $q-\bar{q}$ annihilation process at order- $\alpha_{s}$. The signs of $A_{0}$, $A_{1}(\mu), A_{2}(\nu)$ for the four cases are also listed.

\begin{tabular}{lcccccc}
\hline \hline case & gluon emitted from & $\theta_{1}$ & $\phi_{1}$ & $A_{0}$ & $A_{1}(\mu)$ & $A_{2}(\nu)$ \\
\hline 1 & beam quark & $\beta$ & 0 & + & + & + \\
2 & target antiquark & $\beta$ & $\pi$ & + & - & + \\
3 & beam antiquark & $\pi-\beta$ & 0 & + & - & + \\
4 & target quark & $\pi-\beta$ & $\pi$ & + & + & + \\
\hline \hline
\end{tabular}

that it becomes opposite to the antiquark's momentum vector in the rest frame of $\gamma^{*}$ [Fig. 13(b)]. Since the antiquark's momentum is the same as the target hadron's, the $\hat{z}^{\prime}$ axis is along the direction of $-\vec{p}_{T}$. From Fig. 12, it is evident that $\theta_{1}=\beta$ and $\phi_{1}=0$ in this case. An analogous diagram in which the gluon is emitted from the antiquark in the target hadron is shown in Fig. 13(c). In this case, $\theta_{1}=\beta$ while $\phi_{1}=\pi$. Table IV lists the values of $\theta_{1}$ and $\phi_{1}$ for four cases of different combination of hadron and quark types from which the gluon is emitted [22].

Table IV shows that the sign of $\mu$ could be either positive or negative, depending on which parton and hadron the gluon is emitted from. Hence, one expects some cancellation effects for $\mu$ among contributions from various processes. Each process is weighted by the corresponding density distributions for the interacting partons. At $x_{F} \sim 0$, the momentum fraction carried by the beam parton $\left(x_{B}\right)$ is comparable to that of the target parton $\left(x_{T}\right)$. Therefore, the weighting factors for various processes are of similar magnitude and the cancellation effect could be very significant, resulting in a small value of $\mu$. On the other hand, as $x_{F}$ increases toward $1, x_{B}$ becomes much larger than $x_{T}$. In this case the weighting factors are now dominated by fewer processes, resulting in less cancellation and a larger value of $\mu$. This explains why the $\mu$ parameter exhibits a strong $x_{F}$ dependence in Figs. 7(b), 8(b) and 11.

Table IV also shows that $A_{0}$ and $A_{2}$ have the same sign (positive) for all four cases. This implies the absence of $x_{F^{-}}$ dependent cancellation effect for them. Hence $\lambda$ and $\nu$ have very weak $x_{F}$ dependencies, as shown in Figs. 7(b), 8(b) and 11 . Therefore, the observed strong rapidity dependence for $\mu$ and weak rapidity dependence for $\lambda$ and $\nu$ in pQCD calculation can be nicely described by the geometric picture. In addition, considering the strong $x_{F}$-dependence for the $q_{T}$ distribution of $\mu$ parameters, it will be instructive for the experiments to measure the $q_{T}$ dependence of $\mu$ at several $x_{F}$ regions, instead of integrating over the entire $x_{F}$.

The NLO pQCD expressions of $\lambda$ and $\nu$ as a function of $q_{T}$ in Eq. (6) have been derived based on a geometric picture of collision geometry in the parton level [21,22]. Within the geometric picture, the $A_{0}$ and $A_{2}$ at NLO are equal to $\left\langle\sin ^{2} \theta_{1}\right\rangle$ (Eq. (7)) with $\phi_{1}=0$. Given $q_{T} / Q=$ $\tan \theta_{1}$ or $-\tan \theta_{1}$, the scaling of $A_{0}$ and $A_{2}$ (equivalently $\lambda$ and $\nu$ ) with $q_{T} / Q$ could also be understood.

\section{COMPASS $\pi^{-}+\mathrm{W}$ at $190 \mathrm{GeV}$}
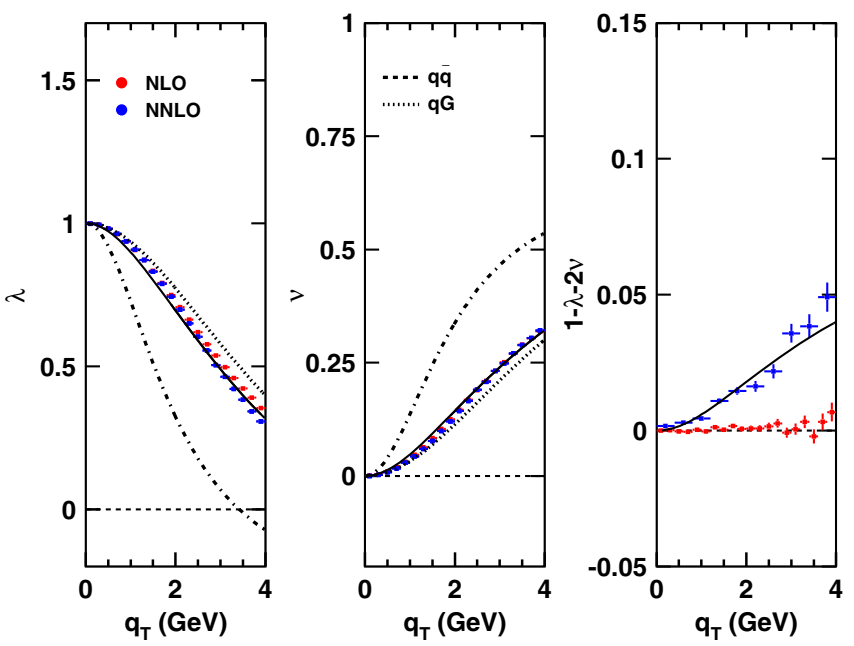

FIG. 14. NLO (red points) and NNLO (blue points) pQCD results of $\lambda, \mu, \nu$ and $1-\lambda-2 \nu$ as a function of $q_{T}$ at the kinematic bin of $5<Q<6 \mathrm{GeV}$ and $0.2<x_{F}<0.4$ for D-Y production off the tungsten target with $190-\mathrm{GeV} \pi^{-}$beam in COMPASS experiment. The NLO pQCD expressions of $q \bar{q}$ and $q G$ processes are denoted by the dotted and dash-dotted lines respectively. The solid curves correspond to the fit results described in the text.

Figure 14 shows both NLO (red points) and NNLO (blue points) pQCD results of $\lambda, \nu$ and $1-\lambda-2 \nu$ as a function of $q_{T}$ at the kinematic bin of $5<Q<6 \mathrm{GeV}$ and $0.2<x_{F}<0.4$ for the COMPASS experiment. The corresponding NLO pQCD expressions of $q_{T}$ dependence for $q \bar{q}$ and $q G$ subprocesses in Eq. (6) are drawn as dotted and dotteddash curves. Assuming the fraction of these two processes is $q_{T}$ independent, a best-fit to the NNLO results of $\lambda$ yields the fraction of $q \bar{q}$ process to be $83 \%$ for the COMPASS experiment. This value is consistent with pQCD results shown in Fig. 9. Applying this relative fraction of two pQCD processes, the NNLO result of $\nu$ could be reasonably well described, as shown in Fig. 14, with the acoplanarity parameter $\left\langle\cos 2 \phi_{1}\right\rangle$, set at 0.94 . The predicted $q_{T}$ distribution of the L-T violation $1-\lambda-2 \nu$ from the NNLO pQCD could be then nicely described as well.

Overall our studies show that salient features of $q_{T} / Q$ scaling and $x_{F}$ dependency for the $\lambda, \nu, \mu$ parameters of fixed-target D-Y experiments evaluated by NLO pQCD as well as the L-T violation $1-\lambda-2 \nu$ from the NNLO pQCD can be nicely understood using the geometric picture.

\section{SUMMARY AND CONCLUSION}

We have presented a comparison of the measurements of the angular parameters $\lambda, \mu, \nu$ and $1-\lambda-2 \nu$ of the D-Y process from the fixed-target experiments with the corresponding results from the NLO and NNLO pQCD calculations. Qualitatively the transverse momentum $\left(q_{T}\right)$ dependence of $\lambda, \mu$ and $\nu$ in the data could be described 
by pQCD. The difference between NLO and NNLO results becomes visible at large $q_{T}$. The L-T violation part $1-\lambda-2 \nu$ remains zero in the NLO pQCD calculation and turns positive in NNLO pQCD. It is contrary to the measured negative values in the pion-induced D-Y experiments NA10 and E615. Data quality, nonperturbative effects such as Boer-Mulders function at low $q_{T}$ and higher-order perturbative QCD at large $q_{T}$ might account for the discrepancy.

From the NLO pQCD calculation, we then present the predictions of the angular parameters as a function of $q_{T}$ in several $Q$ and $x_{F}$ bins for the ongoing COMPASS and SeaQuest experiments. The $\lambda$ and $\nu$ show some mild dependence on $Q$ and a weak $x_{F}$ dependence, while $\mu$ exhibits a pronounced dependence on $x_{F}$. For different $x_{F}$-values, $\lambda$ and $\nu$ are predicted to approximately scale with $q_{T} / Q$.

The $x_{F}$ dependence of the angular parameters is well described by the geometric picture. In particular, the weak rapidity dependencies of the $\lambda$ and $\nu$, and the pronounced rapidity dependency for $\mu$ can be explained by the absence or presence of rapidity-dependent cancellation effects. The occurrence of acoplanarity between the quark plane and the hadron plane $\left(\phi_{1} \neq 0\right)$, for the pQCD processes beyond NLO leads to a violation of the $L-T$ relation. The predicted positive value of $1-\lambda-2 \nu$, or $A_{0}>A_{2}$ when $\phi_{1}$ is nonzero, is consistent with the NNLO pQCD results.

The resummation effect of soft-gluon emission is not taken into account in this work. In the geometric approach, summing over multiple gluon emissions by a single quark line is equivalent to an emission of a single gluon. Therefore, as long as the resummation is only performed for a single quark, the L-T relation will still be satisfied, as shown in Ref. [31]. For a comprehensive pQCD calculation, the resummation effect should be included, especially in the small $q_{T}$ region. We leave it for future investigation.

The NLO and NNLO pQCD calculations should provide a good benchmark for understanding the experimental data of lepton angular distributions of fixed-target D-Y experiments. It is interesting to see many salient features present in $\mathrm{PQCD}$ results can be readily understood by the geometric picture. This intuitive approach could offer some useful insights on the origins of many interesting characteristics of the lepton angular distributions in the forthcoming new precision data from the COMPASS and SeaQuest experiments. Any deviation from the $\mathrm{PQCD}$ results on the L-T violation as well as the $\nu$ parameter would indicate the presence of nonperturbative effects such as the BoerMulders functions. Finally we emphasize the importance of measuring the angular parameters in the D-Y process, which provides a powerful tool to explore the reaction mechanism and parton distributions potentially more sensitively than the D-Y cross sections alone. The measurement of the $q_{T}$ distributions of $\mu$ parameters with $x_{F}$ dependence is suggested, and the pQCD effect should be included in the extraction of nonperturbative Boer-Mulders effect from the data of $\nu$.

\section{ACKNOWLEDGMENTS}

This work was supported in part by the U.S. National Science Foundation and the Ministry of Science and Technology of Taiwan. It was also supported in part by the U.S. Department of Energy, Office of Science, Office of Nuclear Physics under Contract No. DE-AC05-060R23177.
[1] S. D. Drell and T. M. Yan, Phys. Rev. Lett. 25, 316 (1970); Ann. Phys. (N.Y.) 66, 578 (1971).

[2] J. C. Peng and J. W. Qiu, Prog. Part. Nucl. Phys. 76, 43 (2014).

[3] S. Falciano et al. (NA10 Collaboration), Z. Phys. C 31, 513 (1986); M. Guanziroli et al., Z. Phys. C 37, 545 (1988).

[4] J. S. Conway et al. (E615 Collaboration), Phys. Rev. D 39, 92 (1989); J. G. Heinrich et al., Phys. Rev. D 44, 1909 (1991).

[5] W. C. Chang and D. Dutta, Int. J. Mod. Phys. E 22, 1330020 (2013).

[6] A. Baldit et al. (NA51 Collaboration), Phys. Lett. B 332, 244 (1994).

[7] R. S. Towell et al. (E866/NuSea Collaboration), Phys. Rev. D 64, 052002 (2001).

[8] W. C. Chang and J. C. Peng, Prog. Part. Nucl. Phys. 79, 95 (2014).
[9] A. Bacchetta, F. Delcarro, C. Pisano, M. Radici, and A. Signori, J. High Energy Phys. 06 (2017) 081.

[10] D. Boer, Phys. Rev. D 60, 014012 (1999).

[11] M. Aghasyan et al. (COMPASS Collaboration), Phys. Rev. Lett. 119, 112002 (2017).

[12] C. S. Lam and W. K. Tung, Phys. Rev. D 18, 2447 (1978).

[13] C. S. Lam and W. K. Tung, Phys. Rev. D 21, 2712 (1980).

[14] A. Brandenburg, O. Nachtmann, and E. Mirkes, Z. Phys. C 60, 697 (1993).

[15] L. Y. Zhu et al. (Fermilab E866 Collaboration), Phys. Rev. Lett. 99, 082301 (2007); 102, 182001 (2009).

[16] T. Aaltonen et al. (CDF Collaboration), Phys. Rev. Lett. 106, 241801 (2011).

[17] V. Khachatryan et al. (CMS Collaboration), Phys. Lett. B 750, 154 (2015).

[18] G. Aad et al. (ATLAS Collaboration), J. High Energy Phys. 08 (2016) 159. 
[19] R. Gauld, A. Gehrmann-De Ridder, T. Gehrmann, E. W. N. Glover, and A. Huss, J. High Energy Phys. 11 (2017) 003.

[20] M. Lambertsen and W. Vogelsang, Phys. Rev. D 93, 114013 (2016).

[21] J. C. Peng, W. C. Chang, R. E. McClellan, and O. Teryaev, Phys. Lett. B 758, 384 (2016).

[22] W. C. Chang, R. E. McClellan, J. C. Peng, and O. Teryaev, Phys. Rev. D 96, 054020 (2017).

[23] J. C. Peng, D. Boer, W. C. Chang, R. E. McClellan, and O. Teryaev, Phys. Lett. B 789, 356 (2019).

[24] COMPASS-II Proposal, Report No. CERN-SPSC-2010, http://cds.cern.ch/record/1265628.

[25] P. E. Reimer (Fermilab SeaQuest Collaboration), J. Phys. Conf. Ser. 295, 012011 (2011).

[26] G. Altarelli, R. K. Ellis, and G. Martinelli, Nucl. Phys. B157, 461 (1979); J. Kubar-Andre and F. E. Paige, Phys. Rev. D 19, 221 (1979); K. Harada, T. Kaneko, and N. Sakai, Nucl. Phys. B155, 169 (1979); B165, 545(E) (1980).

[27] R. K. Ellis, G. Martinelli, and R. Petronzio, Nucl. Phys. B211, 106 (1983); R. J. Gonsalves, J. Pawlowski, and C. F. Wai, Phys. Rev. D 40, 2245 (1989); P. B. Arnold and M. H. Reno, Nucl. Phys. B319, 37 (1989); B330, 284(E) (1990).

[28] S. Catani, L. Cieri, G. Ferrera, D. de Florian, and M. Grazzini, Phys. Rev. Lett. 103, 082001 (2009); S. Catani and M. Grazzini, Phys. Rev. Lett. 98, 222002 (2007).

[29] K. Melnikov and F. Petriello, Phys. Rev. D 74, 114017 (2006).
[30] D. Boer and W. Vogelsang, Phys. Rev. D 74, 014004 (2006).

[31] E. L. Berger, J. W. Qiu, and R. A. Rodriguez-Pedraza, Phys. Lett. B 656, 74 (2007); Phys. Rev. D 76, 074006 (2007).

[32] DYNNLO v1.5, http://theory.fi.infn.it/grazzini/dy.html.

[33] A. Buckley, J. Ferrando, S. Lloyd, K. Nordstrm, B. Page, M. Rfenacht, M. Schnherr, and G. Watt, Eur. Phys. J. C 75, 132 (2015).

[34] Official LHAPDF6 PDF sets, https://lhapdf.hepforge.org/ pdfsets.html.

[35] J. C. Collins and D. E. Soper, Phys. Rev. D 16, 2219 (1977).

[36] B. Zhang, Z. Lu, B. Q. Ma, and I. Schmidt, Phys. Rev. D 77, 054011 (2008); Z. Lu and I. Schmidt, Phys. Rev. D 81, 034023 (2010); X. Wang, W. Mao, and Z. Lu, Eur. Phys. J. C 78, 643 (2018).

[37] V. Barone, S. Melis, and A. Prokudin, Phys. Rev. D 81, 114026 (2010); V. Barone, S. Melis, and A. Prokudin, Phys. Rev. D 82, 114025 (2010).

[38] J. C. Peng, Eur. Phys. J. Web Conf. 85, 01009 (2015).

[39] Fermilab E1039 Proposal, Report. No. FERMILAB-PROPOSAL-103, http://inspirehep.net/record/1309534.

[40] J. C. Collins, Phys. Rev. Lett. 42, 291 (1979).

[41] R. L. Thews, Phys. Rev. Lett. 43, 987 (1979).

[42] J. Lindfors, Phys. Scr. 20, 19 (1979). 